\title{
Hydrodynamic Analysis of Horizontal-Axis Tidal Current Turbine with Rolling and Surging Coupled Motions
}

\author{
Shu-qi WANG ${ }^{1}, \mathrm{Ke} \mathrm{SUN}^{2}$, Gang XU' ${ }^{1}$, Yong-tao $\mathrm{LIU}^{1}, \mathrm{Xu} \mathrm{BAI}{ }^{1}$
}

\begin{abstract}
The hydrodynamic performance of a turbine with multi-degree of freedom (rotation, rolling, surging) coupled motion in the unbounded uniform flow is analysed by the sliding and dynamic meshes. The effects of the turbine's hydrodynamic load has been studied and illustrated considering the coupled motion of rotation, rolling and surging. Numerical results shows that: 1) the effects of the hydrodynamic load is mainly caused by the surging motion, namely, the instantaneous values of the axial load and power coefficients generate fluctuation with a frequency that is the same as that of the surging and an amplitude that similarly increases in parallel. The effect of the rolling motion on hydrodynamic load will be reflected when its frequency is greater than that of the surging; 2) the calculation formulas of the turbine's axial load and power coefficients has been obtained. The results of the hydrodynamic load calculated by the calculation formula and CFD numerical simulation show good agreements, which verify the calculation formulas. The results of this research can provide relevant data for the hydrodynamic analysis of turbines with multi-degree of freedom motion and verify the structural design and control of the electric output.
\end{abstract}

Key words: Tidal current energy; Horizontal-axis turbine; Multi-degree of freedom motion; Hydrodynamic load; Coupled motion

\section{Introduction}

With the development of the worldwide economy, the demand for energy increases year by year, so more and more countries have begun to consider pursuing clean renewable energy. Among them, tidal current energy [1] does not have significant variation between years and seasons. It also has many other advantages, including its sustainability and predictability and its large energy density, and it does not occupy any land area, so it is viewed very favourably. According to the relationship between the turbine rotation axis and the direction of inflow, tidal current turbines can be divided into vertical-axis turbines and horizontal-axis turbines. The horizontal-axis turbine [2-6] has attracted much attention because of its advantages, such as that the change in the load in the flow field is not very large, and the output of power is stable.

Scholars at home and abroad have carried out a large number of experiments on the horizontal-axis tidal current turbine that can be divided into two broad categories as follows: experiments in which the effects of waves are considered and those in which the effects of waves are not considered. In 2006, the effects of the wing section, taper and hub pitch angle on the power coefficient and cavitation number were studied by Batten [7]. The study found that the phenomenon of cavitation can be minimized or even avoided by selecting an appropriate hub pitch angle or wing section with a higher camber. In 2007, Bahaj [8] tested a three-bladed tidal energy turbine with a diameter of $0.8 \mathrm{~m}$ and profile NACA63-8xx in a cavitation tunnel and towing tank. In the cavitation tunnel experiment, the hub pitch angle of the turbine was set to 15 to 30 degrees, and the flow velocity, to 0.8 to $2 \mathrm{~m} / \mathrm{s}$. In the towing tank experiment, the tip-immersion of the turbine was set to 0.19D to 0.55D (D is the diameter of the turbine), and the yaw angle, to 0 to 30 degrees from the axis. It was observed that the power coefficient of the turbine reaches its peak when the hub pitch angle is 20 degrees. The power coefficient decreases by $10-15 \%$ when the tip-immersion changes from 0.55D to 0.19D because of the influence of the free surface. In 2010, Maganga [9] tested a three-bladed turbine with a diameter of $0.7 \mathrm{~m}$ in a closed circulation water channel. The turbulence levels of inflow ranged from $8 \%$ to $25 \%$; the yaw angle was - 10 to 20 degrees; and the depth below the free surface was 0.94D, 1.37D and 2.04D. The experiment determined that when the inflow has a high turbulence level, the 
efficiency and thrust coefficient decrease by approximately 9\%, and the performance of the turbine decreases significantly with a large yaw angle. These experiments did not consider the effect of waves and mainly studied the effects of the wing section, inflow yaw angle and free surface on the horizontal-axis tidal current turbine hydrodynamic performance. They also did not study the influence on hydrodynamic performance when the turbine generates a swaying movement.

In 2007, Barltrop [10] studied the wave effect on the properties of a tidal current turbine. A horizontal axis turbine with three blades was tested in a towing tank, and the average values of torque and drag were measured in a regular wave. The research results show that, within the scope of a wave period, all average values of the measured parameters were the same as those without a wave, but the instantaneous value changes of the drag and torque are obvious. In 2010, Gallway [11] conducted a similar model test in the tank on a three-blade turbine with a horizontal axis. The result of this experiment was similar to that of Barltrop: the variation in the thrust was approximately $37 \%$, and that of the torque, $35 \%$. This means that the varying load on the blades will significantly accelerate the fatigue of the turbine, posing a challenge to the design of a full-scale tidal turbine. In 2013, Luznik [12], considering the effects of surface gravity waves, tested a three-bladed turbine with and without waves in the United States Naval Academy towing tank. The research results show that under the action of surface gravity waves, the average turbine performance did not appreciably change, but the axial load and instantaneous power of the turbine changed cyclically, and the frequency was consistent with the surface wave frequency. In 2014, Ethan [13] carried out a model test (wave and without wave) on a two-blade horizontal turbine in a towing tank. The diameter of the model was $800 \mathrm{~mm}$, and the immersion depth was 1.3D and 2.25D. The resistance, torque and rotational speed were tested. The test showed that the results at the two different immersing depths were similar, and when a wave existed, the thrust and power experienced periodical changes, and the oscillation frequency was consistent with the wave frequency. Under different combinations of rotation speed and waves, the oscillations of the test power and resistance were $39 \%$ and as high as $79 \%$, respectively. This is a phenomenon that we need to pay attention to. A surface wave was generally adopted in the test, and the effects of the surface wave on the turbine performance had a lot to do with the immersion depth of the blade tip. The turbine was fixed on a trailer or flume without any movement. However, in an actual situation of a floating tidal current power station [14], due to the effect of waves, the floating carrier would generate wave-induced movements that were generally multi-degree of freedom coupled motions, causing the turbine to undergo motion with a floating carrier. Therefore, the hydrodynamic characteristics of the turbine will be affected by the wave and turbine motion. To make the floating tidal current power station run safely and reliably, it is necessary to study the hydrodynamic characteristics of the turbine when it generates movement under the wave condition.

The hydrodynamic analysis of the horizontal-axis turbine in a wave-current field is very complicated, and it is difficult to use CFD software to simulate the wave-current field directly. Hence, we simplify the hydrodynamic problems of the horizontal-axis turbine with multi-degree freedom wave-induced movements and put forward the following two assumptions: 1) the turbine is fixed on a floating carrier with a main shaft, and the floating carrier has a slight simple harmonic motion with wave action; and 2) the inflow velocity is a constant with no influence of waves, which means that we assume that the tip-immersion depth is deep enough. Based on these assumptions, the hydrodynamic problems of the horizontal-axis turbine with wave motion can be simplified as problems of a turbine with multi-degree of freedom simple harmonic motion in a constant tidal current.

The literature [15] reports on the hydrodynamic load of a horizontal-axis turbine with coupled motion of surging and rotation, and found that the hydrodynamic load is affected by the frequency and amplitude of surging. On the basis of the literature and CFD, this paper studied the hydrodynamic load of a horizontal-axis turbine in unbounded uniform flow using sliding and dynamic meshes when it generated rotation, rolling and surging multi-degree of freedom coupled motion; analysed the result of the CFD when the turbine only generated rotation 
and a single degree of swaying motion (surging and rolling); an obtained the calculation formula of axial load and energy efficiency when the turbine generates rotation, rolling and surging coupled motion. The research focus on the effect laws of the turbine's hydrodynamic load under the condition of rolling and surging coupled motion of co-frequency and different frequency at a constant rotation speed. The result provides relevant data for studying the wave motion response of a floating carrier and designing the turbine structure and control system of the electrical energy.

Nomenclature

\begin{tabular}{c|l}
\hline Symbols & \\
\hline$U$ & Tidal current flow speed (unit: $\mathrm{m} / \mathrm{s}$ ) \\
$u$ & Swaying velocity (unit: $\mathrm{m} / \mathrm{s}$ ) \\
$\omega_{T}$ & Rotation speed of turbine (unit: rad/s) \\
$Z_{z d}$ & Surging displacement (unit: $\mathrm{m}$ ) \\
$\omega_{z d}$ & Surging frequency (unit: rad/s) \\
$A_{z d}$ & Surging amplitude (unit: $\mathrm{m}$ ) \\
$\theta_{h y}$ & Rolling angle (unit: degree) \\
$\omega_{h y}$ & Rolling frequency (unit: rad/s) \\
$A_{h y}$ & Rolling amplitude (unit: degree) \\
$D$ & Diameter of turbine (unit: $\mathrm{m}$ ) \\
$R$ & Radius of turbine (unit: $\mathrm{m}$ ) \\
$N$ & Number of blades \\
$F_{Z}$ & Axial force (unit: N) \\
$M_{h y}$ & Roll moment (unit: N/m) \\
$M_{Z}$ & Axial torque (unit: N/m) \\
$\rho$ & Density of the inflow water (unit: $\mathrm{kg} / \mathrm{m}^{3}$ ) \\
$\varepsilon$ & initial phase (unit: rad) \\
\hline
\end{tabular}

\section{The fitting formula of hydrodynamic loads on turbine}

In the working condition of the horizontal-axis turbine, it is in normal operation and generates a single degree of freedom swaying movement. The hydrodynamic load of the turbine is affected by the rotation and swaying frequencies. According to the features of the turbine hydrodynamic load, we select appropriate expressions to fit the hydrodynamic load of the horizontal-axis turbine. The following will introduce a specific calculation method of the fitting formulas when the horizontal-axis turbine is in the working condition. The uniform inflow velocity is $U$, and the $\mathrm{Z}$ axis normal direction is the direction of the inflow velocity; the velocity of the turbine swaying is $u$; the angle of the blades' positions is $\theta(t)=\omega_{T} t+\varepsilon$. Set $\bar{u}_{y d}$ and $\bar{a}_{y d}$ as the dimensionless swaying velocity and acceleration, respectively; the hydrodynamic load coefficient of the turbine with swaying movement is $C_{y d}$. When the turbine generates micro-amplitude movement, we can divide the hydrodynamic force into a uniform current hydrodynamic force, damping resistance and added mass force, with the formula as follows

$$
C_{y d}=C_{y d}^{F}+n_{y d} \bar{u}_{y d}+m_{y d} \bar{a}_{y d}
$$

Where, $C_{y d}^{F}$ is the hydrodynamic force coefficient without swaying movement, $n_{y d}$ and $m_{y d}$ are the swaying damping and added mass coefficients respectively.

According to the characteristics of the hydrodynamic loads on horizontal-axis turbine, $C_{y d}^{F}, n_{y d}$ and $m_{y d}$ in Formula (1) can be expanded into the form of trigonometric series:

$$
C_{y d}^{F}=C_{y d}^{0}+\sum_{k=1}^{\infty} C_{y d}^{k} \sin \left(k N \theta(t)+\psi_{y d}^{k}\right)
$$




$$
\begin{aligned}
& n_{y d}=n_{y d}^{0}+\sum_{k=1}^{\infty} n_{y d}^{k} \sin \left(k N \theta(t)+\psi_{u, y d}^{k}\right) \\
& m_{y d}=m_{y d}^{0}+\sum_{k=1}^{\infty} m_{y d}^{k} \sin \left(k N \theta(t)+\psi_{a, y d}^{k}\right)
\end{aligned}
$$

Therefore, the turbine hydrodynamic load coefficient curve can be represented by the following formula:

$$
\begin{aligned}
C_{y d}= & C_{y d}^{0}+\sum_{k=1}^{\infty} C_{y d}^{k} \sin \left(k N \theta(t)+\psi_{y d}^{k}\right) \\
& +\left\{n_{y d}^{0}+\sum_{k=1}^{\infty}\left[n_{y d}^{k} \sin \left(k N \theta(t)+\psi_{u, y d}^{k}\right)\right]\right\} \bar{u}_{y d} \\
& +\left\{m_{y d}^{0}+\sum_{k=1}^{\infty}\left[m_{y d}^{k} \sin \left(k N \theta(t)+\psi_{\mathrm{a}, \mathrm{yd}}^{k}\right)\right]\right\} \bar{a}_{y d}
\end{aligned}
$$

Because the flow field of the horizontal axis turbine is very complicated with a dynamic stall and wake, it is difficult to solve for these coefficients (damping coefficient and added mass coefficient) directly. However, the turbine hydrodynamic load coefficient $C_{y d}$ can be obtained by experiment or CFD simulation, so this paper is based on the least squares method to fit the hydrodynamic load time-varying curves according to Formula (5) and obtain different roll hydrodynamic coefficients with different roll parameters.

\section{CFD numerical value}

\section{0 \\ 3.1 Building calculation model}

The blade used by the calculation model was designed by the Institute of Ocean Renewable Energy System (IORES) at Harbin Engineering University (HEU), China. Each cross-section aerofoil is S809, The chord length and twist angle change with the radius as shown in Table 1.

Table 1 Parameters of turbine

\begin{tabular}{c|c|c}
\hline Radius(mm) & Chord(mm) & Pitch(degree) \\
\hline 70 & 90.01 & 26.2 \\
90 & 84.96 & 19.9 \\
110 & 80.11 & 15.5 \\
130 & 75.46 & 12.2 \\
150 & 71.01 & 9.8 \\
170 & 66.76 & 7.9 \\
190 & 62.71 & 6.3 \\
210 & 58.86 & 5.0 \\
230 & 55.21 & 4.0 \\
250 & 51.76 & 3.1 \\
270 & 48.51 & 2.3 \\
290 & 45.46 & 1.6 \\
310 & 42.61 & 1.1 \\
330 & 39.96 & 0.5 \\
350 & 37.51 & 0.1 \\
\hline
\end{tabular}

The diameter (D) of the turbine calculation model is $0.7 \mathrm{~m}$; the number of blades is 2; both end hubs are hemispherical with a diameter of 0.1D, as shown in Figure 1(a). To adequately simulate the characteristics of the 
computational domain is cuboid, with a square bottom. The side length of the square is $10 \mathrm{D}$, and the length of the cuboid is $20 \mathrm{D}$. The distance between the turbine rotational plane and the entrance boundary is $5 D$. In the simulation of the horizontal-axis turbine with considered coupled motion, not only the rotational motion but also the rolling $\left(\theta_{h y}=A_{h y} \sin \left(\omega_{h y} t\right)\right)$ and surging $\left(Z_{z d}=A_{z d} \sin \left(\omega_{z d} t\right)\right)$ coupled motion needs to be considered in the numerical

simulation. In the simulation of a turbine with rotational and rolling coupled motion, the rotational motion and roll motion are simulated at the same time. In the simulation of a turbine with rotational and surging coupled motion, the rotational motion and surging motion are simulated at the same time. To avoid mesh quality reduction in the process of calculation, the whole computational domain should be divided into several blocks, as shown in Table 2 .

\begin{tabular}{|c|c|c|c|}
\hline Calculation condition & \multicolumn{3}{|c|}{ Division of computational domain } \\
\hline $\begin{array}{l}\text { rotation, rolling and surging coupled motion } \\
\text { rotation and rolling coupled motion } \\
\text { rotation and surging coupled motion }\end{array}$ & $\begin{array}{l}\text { rotation domain } \\
\text { rotation domain } \\
\text { rotation domain }\end{array}$ & $\begin{array}{l}\text { rolling domain } \\
\text { rolling domain } \\
\text { surging domain }\end{array}$ & $\begin{array}{c}\text { surging domain deformation domain } \\
\text { static domain } \\
\text { deformation domain }\end{array}$ \\
\hline
\end{tabular}

In the simulation of rotation, rolling and surging coupled motion, the entire computational domain is divided into four parts as follows: rotation domain, rolling domain, surging domain and deformation domain, as shown in Figure 1. The rotation domain is within the rolling domain, the rolling domain is within the surging domain, and the surging domain is next to the deformation domain. The distance between the turbine rotation axis and the centre of the roll is $0.4 \mathrm{~m}$. In the simulation of the rotation and rolling coupled motion, the entire computational domain is divided into three parts as follows: rotation domain, rolling domain and static domain, as shown in Figure 2. The rotation domain is within the rolling domain, and the rolling domain is within the static domain. The distance between the turbine rotation axis and the centre of the roll is $0.4 \mathrm{~m}$. In the simulation of the rotation and surging coupled motion, the entire computational domain is divided into three parts as follows: rotation domain, surging domain and deformation domain, as shown in Figure 3. The rotation domain is within the surging domain, and the surging domain is next to the deformation domain.

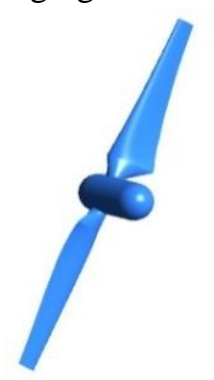

(a) turbine

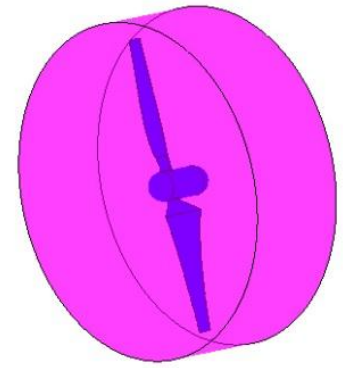

(b) rotation domain

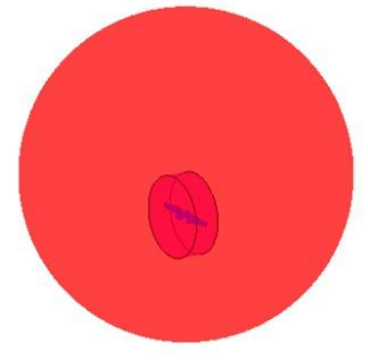

(c) rolling domain

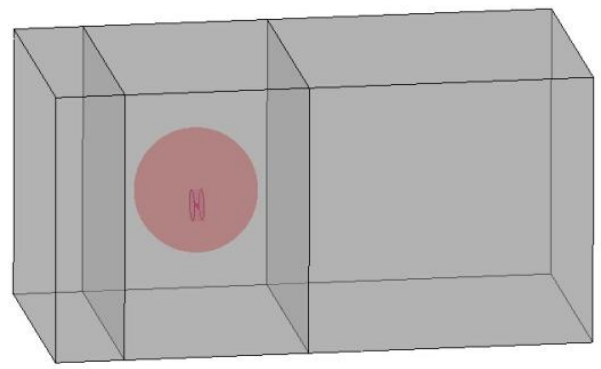

(d) entire computational domain

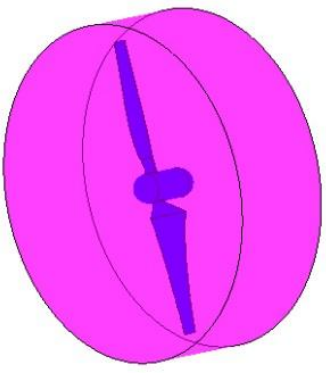

(a) rotation domain

Fig. 1 Calculation model of rotation, rolling and surging coupled motion

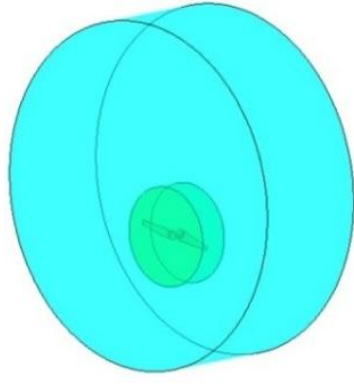

(b) rolling domain

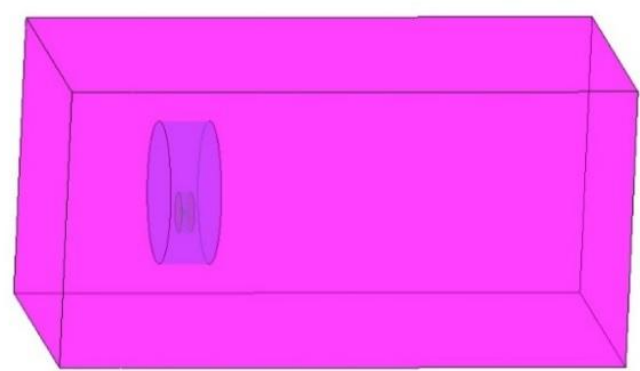

(c) entire computational domain

Fig. 2 Calculation model of rotation and rolling coupled motion 


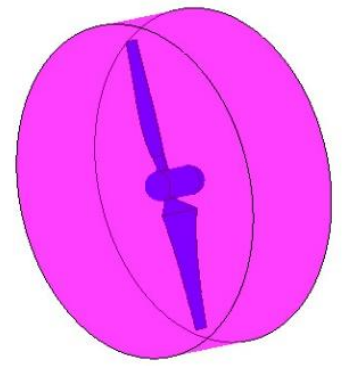

(a) rotation domain

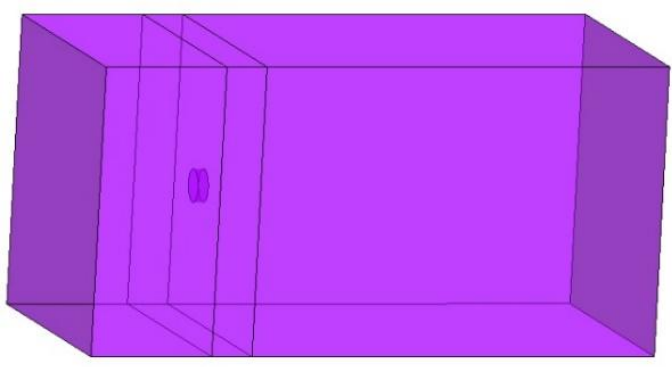

(b) entire computational domain

Fig. 3 Calculation model of rotation and surging coupled motion

\subsection{Meshing}

The computational domain requires discretization when it is built, namely, meshing. Meshing is a very important element of the numerical simulation technique as the grid quality directly affects the accuracy and efficiency of the numerical calculation. The meshing of the entire computational domain adopts the methods of structured grid and unstructured grid. Because the shape of the rotation domain for the turbine is complex, it is difficult to achieve a high-quality structured grid without simplifying model. Therefore, we adopt an unstructured grid; the rolling, surging, deformation and static domains have regulation shapes, so adopting a structured grid is beneficial to improving the calculation efficiency. Referencing [16], the first floor height of the surface grid of the turbine is $0.0005 \mathrm{~m}$, and the $\mathrm{y}+$ ranges in $1 \sim 48$. In the simulation of rotation, rolling and surging coupled motion, the total number of grid units is 2.38 million, as shown in Figure 4. In the simulation of rotation and rolling coupled motion, the total number of grid units is 2.36 million, as shown in Figure 5. In the simulation of rotation and surging coupled motion, the total number of grid units is 2.04 million, as shown in Figure 6.
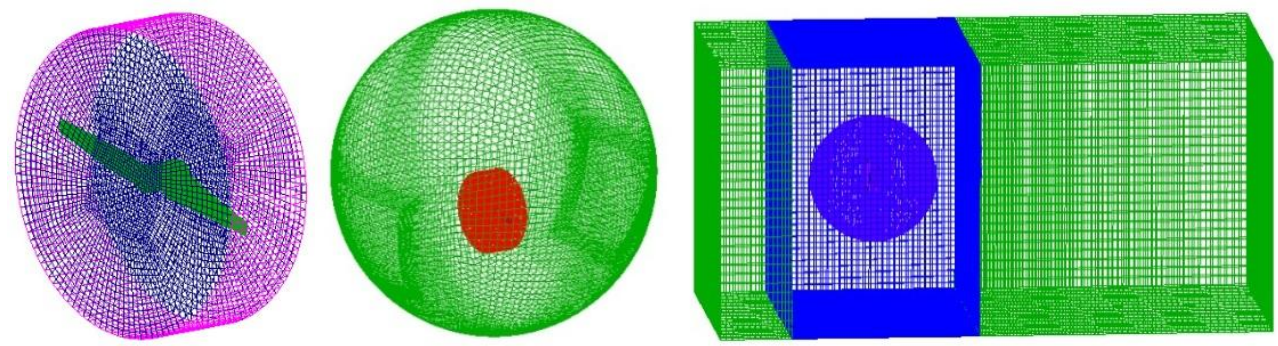

Fig. 4 Grid model of rotation, rolling and surging coupled motion
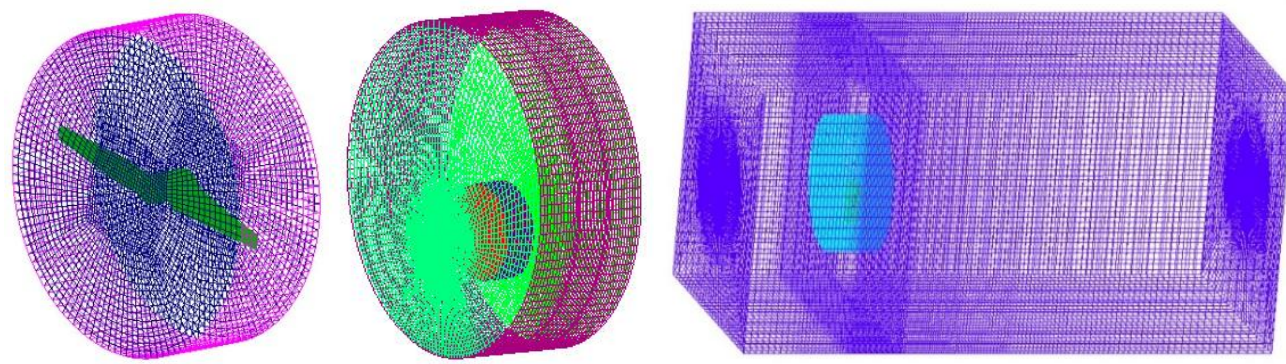

Fig. 5 Grid model of rotation and rolling coupled motion
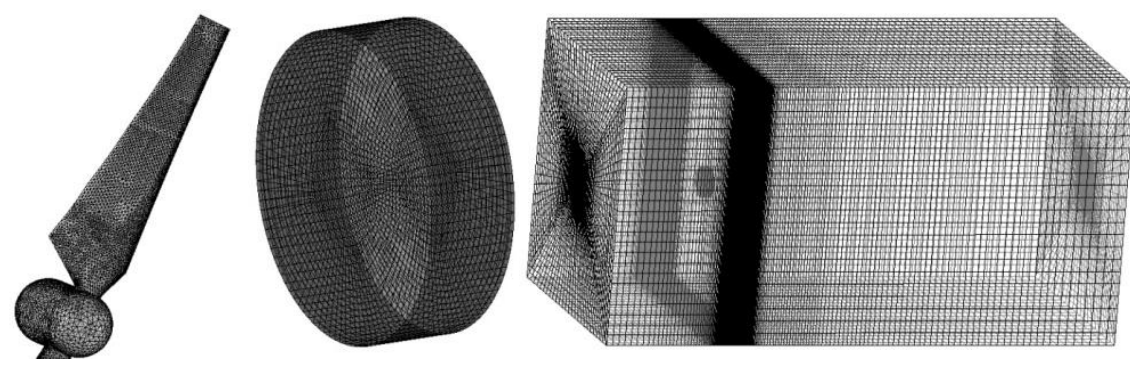

Fig. 6 Grid model of rotation and surging coupled motion 


\subsection{Boundary condition}

Because of the multi-degree of freedom coupled motion of the turbine, we adopt the movement form of the combination of the sliding and dynamic meshes: the dynamic mesh involves only translation, stretching and compression in the inflow direction, without involving the warping of the mesh, whereas the sliding mesh is used for the entire circuitry (rotation motion and rolling motion) of the mesh, so the initial quality of the mesh in the computational process is guaranteed. The setting strategies of rotation, rolling and surging coupled motion are as follows. The surging domain is given with surging motion in the form of a subdomain; the rolling domain is given with roll angular speed, with surging motion in the form of a subdomain; the rotation domain is given with roll angular speed, with rotation and surging motion in the form of a subdomain; and the deformation domain is next to the surging domain, and because of the complete translation of the surging domain, the mesh will generate stretching and compression in the direction of inflow, but without the warping of the mesh. The setting strategies of the rotation and rolling coupled motion are as follows. The rolling domain and rotation domain are given with roll angular speed together; the rotation domain is also given with rotation in the form of a subdomain, which means that the rotation domain rotates and rolls with the roll domain, whereas the whole mesh of the static domain is static. The setting strategies of rotation and surging coupled motion are as follows. The surging domain is given with surging motion in the form of a subdomain; the rotation domain is given with rotation angular speed, with surging motion in the form of a subdomain; the deformation domain is next to surging domain, and the mesh will generate stretching and contraction in the direction of the inflow.

The basic boundary condition settings of the CFD numerical simulation are as follows: set the atmosphere as the reference pressure; the inlet boundary (Inlet) is a velocity entry, given a uniform flow velocity and turbulence parameters; the outlet boundary (Outlet) is a pressure outlet, with the relative pressure set to 0; the sides of the computational domain are set as free slip walls (Free slip wall); the turbine surface is a non-slip wall (No slip wall); the SST model is adopted as a turbulent model, and the transient solver is used. The time period during which the blade rotates $3^{\circ}$ is adopted as the time step of the entire simulation.

\section{Analysis of turbine loads}

To validate the numerical simulation accuracy of the horizontal axis turbine, the turbine is simulated in uniform flow with a constant rotation motion under the same grid model as in Figure 4. For the convenience of subsequent data analysis, we explain the expressions of the correlation coefficient as follows

Power coefficient: $C_{P}=\frac{M_{Z} \omega_{T}}{\frac{1}{2} \rho U^{3} \pi R^{2}}$

$$
\text { Axial force coefficient: } C_{Z}=\frac{F_{Z}}{\frac{1}{2} \rho U^{2} \pi R^{2}}
$$

Tip speed ratio: $\lambda=\frac{\omega_{T} R}{U}=\frac{\pi n R}{30 U}$

Rolling moment coefficient: $C_{T h y}=\frac{M_{h y}}{\frac{1}{2} \rho U^{2} \pi R^{3}}$

In numerical simulation process, we set the atmosphere as the reference pressure; the inlet boundary is a velocity entry, set the velocity as $1.5 \mathrm{~m} / \mathrm{s}$ and turbulence intensity as $5 \%$, the velocity is along the axial direction of the turbine; the drift angle is 0 ; the outlet boundary is a pressure outlet, with the relative pressure set to 0 ; only in a rotating field the rotation speed is given; the sides of the computational domain are set as free slip walls; the turbine 
surface is a non-slip wall; the SST model is adopted as a turbulent model, and the transient solver is used. The time period during which the blade rotates $3^{\circ}$ is adopted as the time step of the entire simulation. The calculated values of the power and axial load coefficients are compared with experimental results that were tested in the towing tank of HEU, as shown in Figure 7. From the figure, we can see that the calculation values and the experimental values of these two parameters are in good accordance for most of the TSR values, except when the speed ratio is around 3 . In fact, generally, the speed ratio is optimum when the energy utilization ratio is the maximum, and in the blade design process, the optimal speed ratio is corresponding to the optimal angle of attack. When the speed ratio is larger than the optimal one and the impeller rotates, the blade section airfoil's angle of attack is smaller than the optimal one and the flow is not separated, which belongs to the problem of flows around with small angle of attack. In such case, the calculation results agree with the experimental results better and more accurate. However, when the speed ratio is less than the optimal one, the blade section airfoil's angle of attack is larger than the optimal one and the flow is separated. Especially when the speed ratios is further reduced, and even the stall phenomenon occurs, so that there is a certain deviation between the calculation and the experimental results.

Overall, according to the numerical simulation of horizontal axis turbine using the CFD method either from the load of the turbine, or from the efficiency of the turbine, the calculated and experimental results are in good agreement, which verified the accuracy and feasibility of the simulation method used in this paper to study the hydrodynamic performance of the horizontal axis turbine. When the turbine generates rotation, rolling and surging coupled motion, the turbine hydrodynamic performance is associated with not only the relevant parameter of rolling motion but also the relevant parameter of surging motion. Hence, the research will analyse the turbine's hydrodynamic performance and load under the condition of the rolling and surging coupled motion of co-frequency and different frequency at a constant rotation speed.

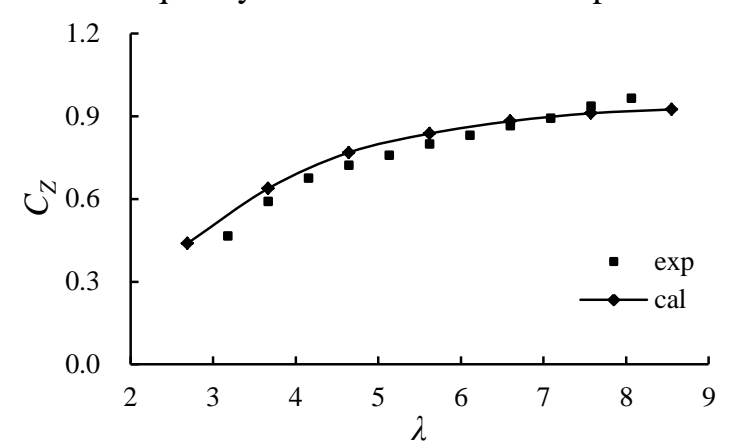

(a) Axial force coefficient

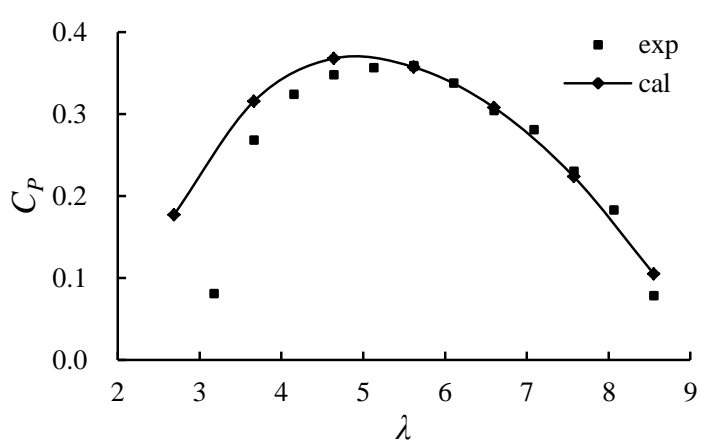

(b) Power coefficient

Fig. 7 Comparison between calculated and experimental values

\subsection{Co-frequency coupled motion}

To study the hydrodynamic characteristics of a horizontal-axis turbine with rotation, rolling and surging co-frequency coupled motion, we set the turbine rotation around the optimum rotational speed $(230 \mathrm{r} / \mathrm{min})$. The rolling amplitude is 15 degrees, the surging amplitude is $0.175 \mathrm{~m}$, and the inlet velocity is $1.5 \mathrm{~m} / \mathrm{s}$. We choose three different frequencies $(0.4 \mathrm{rad} / \mathrm{s}, 1.2 \mathrm{rad} / \mathrm{s}$, and $2 \mathrm{rad} / \mathrm{s})$ to calculate and compare the result with the calculation values when the turbine only generates rotation and rolling coupled motion or rotation and surging coupled motion. Figure 8 shows the axial load coefficient, power coefficient and rolling moment coefficient of the turbine comparison curve with time under different frequencies. The numbers in the graphic legends represent frequencies, with units of rad/s. 2, 1.2 and 0.4 are the calculation results when the turbine generates rotation, rolling and surging coupled motion; zd2, zd1.2 and $\mathrm{zd} 0.4$ are the calculation results when the turbine generates only rotation and 
surging coupled motion; and hy2, hy1.2 and hy0.4 are the calculation results when the turbine only generates rotation and rolling coupled motion.

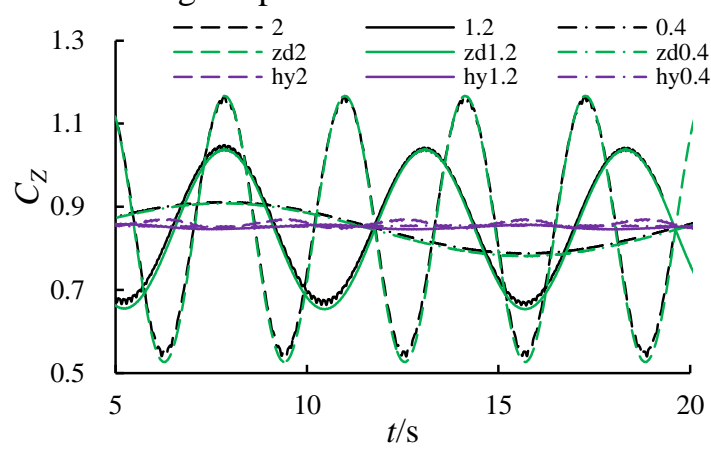

(a) Axial force coefficient

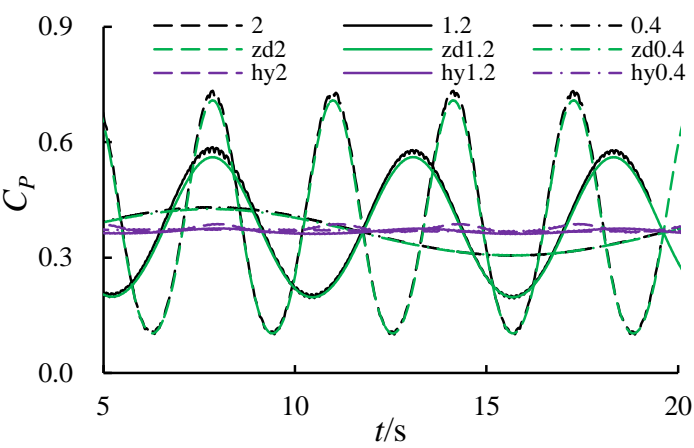

(b) Power coefficient

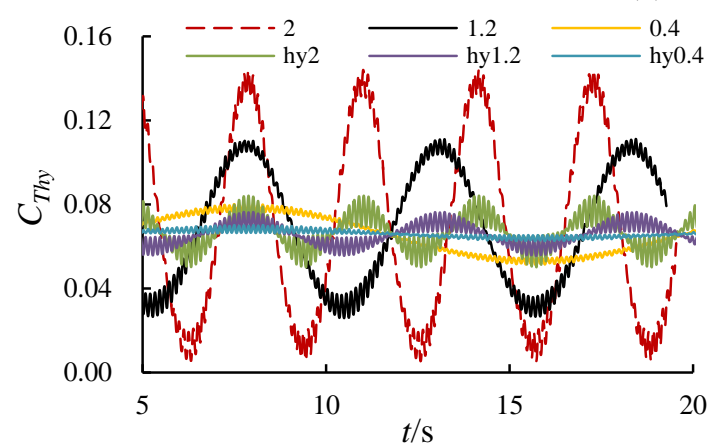

(c) Roll moment coefficient

Fig. 8 Calculated values of co-frequency coupled motion

As shown in Figure 8, when the turbine only generates rotation and surging coupled motion, the axial load coefficient and power coefficient generate clear periodic fluctuations with the turbine surging; the fluctuation frequency is consistent with the surging frequency, and the fluctuation amplitude increases as the surging frequency increases. When the turbine only generates rotation and rolling coupled motion, the axial load coefficient, power coefficient and rolling moment coefficient have fluctuations; the fluctuation frequency is consistent with the rolling frequency; the fluctuation amplitude increases with the rolling frequency, but it is clearly less than the fluctuation amplitude when the turbine generates rotation and surging coupled motion. During the rotation of the turbine, the rolling moment will generate micro-amplitude fluctuation. The frequency of the micro-amplitude fluctuation is double the rotational frequency for a 2-blade turbine, and the maximum amplitude increases with the rolling frequency. When the turbine generates rotation, rolling and surging coupled motion, the axial load coefficient and power coefficient calculation result has an obvious fluctuation; the fluctuation frequency is consistent with the surging (rolling) frequency, and the fluctuation amplitude increases with the frequency; the axial load coefficient and power coefficient calculation results are basically similar to the calculation results when the turbine only generates rotation and surging coupled motion, with only a slight discrepancy in the vicinity of the minimum (axial force coefficient) and maximum (power coefficient) of the fluctuation, so the effect of the rolling motion can almost be ignored. The rolling moment coefficient calculation value is greater than the value when the turbine only generates rotation and rolling coupled motion, and the fluctuation amplitude increases with the frequency. This means that surging motion has a significant impact on the calculation results of the rolling motion. During the process of rotation and co-frequency rolling and surging coupled motion of the turbine, the change rule of the hydrodynamic load is mainly caused by the surging motion.

\subsection{Different frequency coupled motion}

To study the horizontal-axis turbine hydrodynamic characteristics when it generates rotation and rolling and surging different frequency coupled motion, the fixed rotational speed of the turbine is $230 \mathrm{r} / \mathrm{min}$, the rolling 
amplitude is 15 degrees and the surging amplitude is $0.175 \mathrm{~m}$, choosing two different frequency combinations (rolling frequency of $0.4 \mathrm{rad} / \mathrm{s}$ and surging frequency of $2 \mathrm{rad} / \mathrm{s}$, rolling frequency of $1.2 \mathrm{rad} / \mathrm{s}$ and surging frequency of $0.4 \mathrm{rad} / \mathrm{s}$ ) to calculate. The calculation results of the axial load coefficient, power coefficient and rolling moment coefficient are shown in Figure 9. The numbers in the graphic legends represent frequencies, in units of rad/s. zd0.4-hy2 and zd2-hy0.4 are the calculation results when the turbine generates rotation, rolling (hy) and surging (zd) coupled motion, $\mathrm{zd} 2$ and $\mathrm{zd} 0.4$ are the calculation results when the turbine only generates rotation and surging coupled motion, and hy 2 and hy0.4 are the calculation results when the turbine only generates rotation and rolling coupled motion.

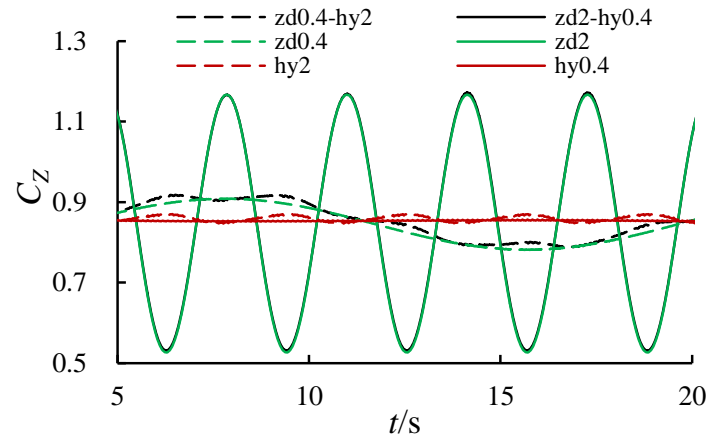

(a) Axial force coefficient

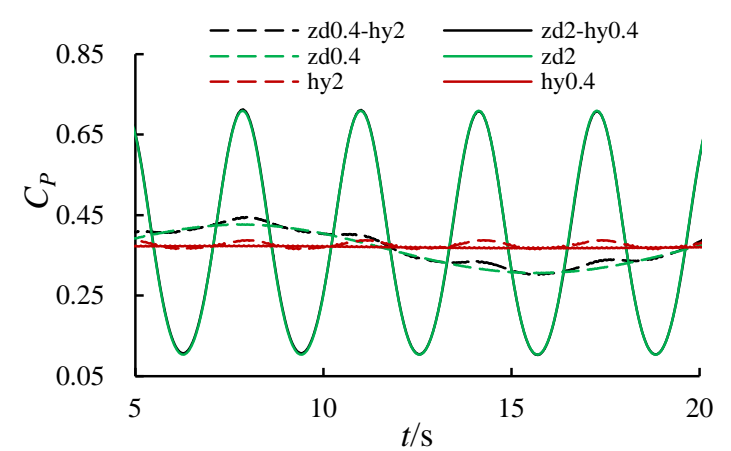

(b) Power coefficient

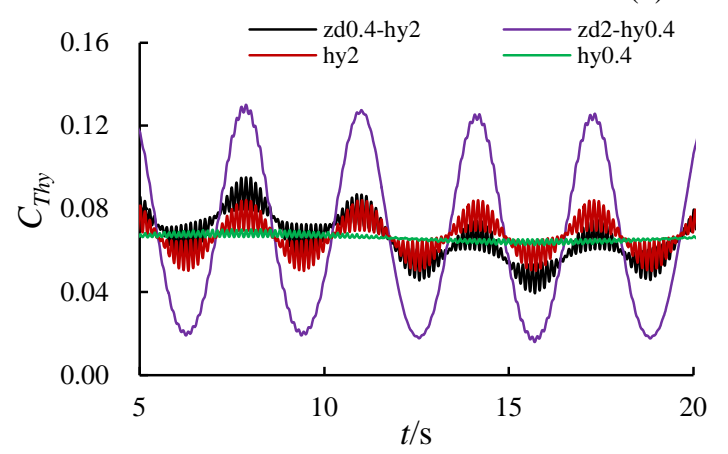

(c) Roll moment coefficient

Fig. 9 Calculated values of different frequencies

In Figure 9, for the axial load coefficient and power coefficient: when surging frequency is higher ( $2 \mathrm{rad} / \mathrm{s})$ and the rolling frequency is lower $(0.4 \mathrm{rad} / \mathrm{s})$, the conclusion is the same as in section 3.1, namely, that the calculation results when the turbine generates rotation, rolling and surging coupled motion and the calculation that results when the turbine only generates rotation and surging coupled motion are basically the same, but when the surging frequency is lower $(0.4 \mathrm{rad} / \mathrm{s})$ and the rolling frequency is higher $(2 \mathrm{rad} / \mathrm{s})$, the calculation results of generating rotation, rolling and surging coupled motion generate a low-frequency fluctuation that is based on the surging frequency and a high-frequency fluctuation that is based on the rolling frequency. For the rolling moment coefficient, when the surging frequency is higher ( $2 \mathrm{rad} / \mathrm{s})$ and the rolling frequency is lower, the fluctuation amplitude of the calculation value when the turbine generates rotation, rolling and surging coupled motion is greater than that when it only generates rotation and rolling coupled motion. When the rolling frequency is higher ( $2 \mathrm{rad} / \mathrm{s}$ ) and the surging frequency is lower $(0.4 \mathrm{rad} / \mathrm{s})$, the fluctuation amplitude of the calculation value when the turbine generates rotation, rolling and surging coupled motion is slightly more than that when it only generates rotation and rolling coupled motion, and the rolling moment coefficient shows a low-frequency fluctuation that is based on the surging frequency and rolling frequency and a high-frequency fluctuation that is based on the rotation frequency of the turbine. When the turbine generates rotation, rolling and surging coupled motion, the change rules of the hydrodynamic load are mainly caused by surging motion, but when the rolling frequency is much higher than the surging frequency, the effect of the rolling motion will be reflected. 


\section{Fitting analysis of hydrodynamic load}

\subsection{Fitting analysis of hydrodynamic load in rotation and surging coupled motion}

The hydrodynamic load fitting formula of the horizontal-axis turbine under the condition of rotation and single degree of freedom swaying motion, which is established in section 2, and the CFD numerical simulation results of the turbine rotation and the surging coupled motion, which is based on the least squares method theory, can be used to solve various coefficients of the fitting formula. The basic parameters of surging motion are defined as follows:

$$
\text { Dimensionless surge velocity: } \bar{u}_{z d}=\frac{A_{z d} \omega_{z d}}{U} \cos \left(\omega_{z d} t\right)
$$

Dimensionless surge acceleration: $\bar{a}_{z d}=-\frac{A_{z d} \omega_{z d}{ }^{2} R}{U^{2}} \sin \left(\omega_{z d} t\right)$

Axial load coefficient progression:

$$
\begin{aligned}
C_{Z} & =C_{Z z d}^{F}+n_{F z d} \bar{u}_{z d}+m_{F z d} \bar{a}_{z d} \\
& =C_{Z z d}^{0}+\sum_{k=1}^{\infty} C_{Z z d}^{k} \sin \left(k N \theta(t)+\psi_{F z d}^{k}\right) \\
& +\left\{n_{F z d}^{0}+\sum_{k=1}^{\infty}\left[n_{F z d}^{k} \sin \left(k N \theta(t)+\psi_{F u z d}^{k}\right)\right]\right\} \bar{u}_{z d} \\
& +\left\{m_{F z d}^{0}+\sum_{k=1}^{\infty}\left[m_{F z d}^{k} \sin \left(k N \theta(t)+\psi_{F a z d}^{k}\right)\right]\right\} \bar{a}_{z d}
\end{aligned}
$$

Power coefficient progression:

$$
\begin{aligned}
C_{P} & =C_{P_{z d}}^{F}+n_{P_{z d}} \bar{u}_{z d}+m_{P_{z d}} \bar{a}_{z d} \\
& =C_{P_{z d}}^{0}+\sum_{k=1}^{\infty} C_{P_{z d}}^{k} \sin \left(k N \theta(t)+\psi_{P_{z d d}}^{k}\right) \\
& +\left\{n_{P_{z d}}^{0}+\sum_{k=1}^{\infty}\left[n_{P_{z d}}^{k} \sin \left(k N \theta(t)+\psi_{P_{u z d}}^{k}\right)\right]\right\} \bar{u}_{z d} \\
& +\left\{m_{P_{z d d}}^{0}+\sum_{k=1}^{\infty}\left[m_{P_{z d}}^{k} \sin \left(k N \theta(t)+\psi_{P a z d}^{k}\right)\right]\right\} \bar{a}_{z d}
\end{aligned}
$$

Utilizing least squares fitting on power coefficients of different frequencies and axial load coefficients, the coefficients of the fitting formula can be calculated, as shown in Table 3 and Table 4; only the constant and first-order terms are given in the tables.

Table $3 C_{z}$ expansion coefficient table (surging amplitude $0.175 \mathrm{~m}$, rotational speed $230 \mathrm{r} / \mathrm{min}$ )

\begin{tabular}{cccccccccc}
\hline$\omega_{z d}$ & $C_{Z z d}^{0}$ & $C_{Z z d}^{1}$ & $\psi_{F z d}^{1}$ & $n_{F z d}^{0}$ & $n_{F z d}^{1}$ & $\psi_{F u z d}^{1}$ & $m_{F z d}^{0}$ & $m_{F z d}^{1}$ & $\psi_{F a z d}^{1}$ \\
\hline 0.4 & 0.845 & 0.0000 & 58.59 & -1.353 & 0.0029 & 5.67 & -0.147 & 0.0098 & -143.98 \\
0.8 & 0.845 & 0.0001 & -167.84 & -1.364 & 0.0002 & -84.56 & -0.080 & 0.0003 & -0.92 \\
1.2 & 0.846 & 0.0001 & -161.51 & -1.367 & 0.0004 & 179.27 & -0.060 & 0.0010 & 1.44 \\
1.6 & 0.846 & 0.0001 & -164.60 & -1.366 & 0.0001 & 133.15 & -0.053 & 0.0003 & 112.56 \\
2.0 & 0.848 & 0.0001 & -153.70 & -1.369 & 0.0000 & -121.55 & -0.053 & 0.0008 & 54.16 \\
\hline \multicolumn{7}{c}{ Table 4 $C_{P}$} & expansion coefficient table (surging amplitude $0.175 \mathrm{~m}$, rotational speed 230 r/min) \\
\hline$\omega_{z d}$ & $C_{P z d}^{0}$ & $C_{P z d}^{1}$ & $\psi_{P z d}^{1}$ & $n_{P z d}^{0}$ & $n_{P_{z d}}^{1}$ & $\psi_{P u z d}^{1}$ & $m_{P_{z d}}^{0}$ & $m_{P z d}^{1}$ & $\psi_{P a z d}^{1}$ \\
\hline 0.4 & 0.365 & 0.0000 & 27.83 & -1.289 & 0.0014 & 15.99 & -0.215 & 0.0109 & -117.51 \\
0.8 & 0.368 & 0.0002 & -155.77 & -1.289 & 0.0002 & -57.88 & -0.080 & 0.0009 & 89.86 \\
1.2 & 0.372 & 0.0001 & -172.27 & -1.290 & 0.0003 & 166.84 & -0.079 & 0.0013 & -90.55 \\
1.6 & 0.379 & 0.0000 & 73.11 & -1.274 & 0.0000 & 143.08 & -0.467 & 0.0014 & 170.57 \\
2.0 & 0.388 & 0.0002 & 101.31 & -1.286 & 0.0004 & 70.97 & -0.044 & 0.0028 & -35.62 \\
\hline
\end{tabular}

It can be seen from Table 3 and Table 4 that the constant term coefficient is far greater than the first-order term 
coefficients. The values of $C_{Z z d}^{0}$ and $C_{P_{z d}}^{0}$ are basically the same as those of $C_{Z}$ and $C_{P}$ when the turbine generates only rotation motion. According to the correlation coefficients of Table 3 and Table 4, the axial load coefficient and power coefficient when the turbine generates rotation and surging coupled motion can be obtained by eq. (12) and (13). To verify the accuracy of the fitting formula, the axial load coefficient and power coefficient when the surging frequency is $1.2 \mathrm{rad} / \mathrm{s}$ is calculated and compared with the CFD calculation value, as shown in Figure 10. Cal-CFD is the CFD calculation value in the figure, and cal-EXP is the calculation value by the fitting formula. In Figure 10, the CFD calculation value basically agrees with the calculation value by the fitting formula, which verifies the accuracy of the fitting formula.

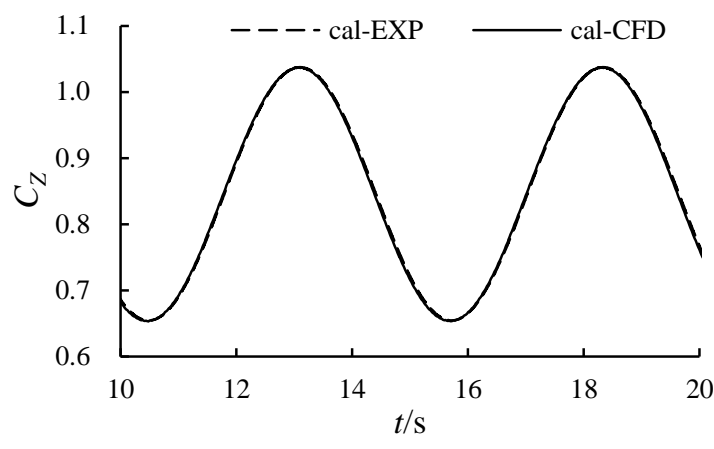

(a) Axial force coefficient

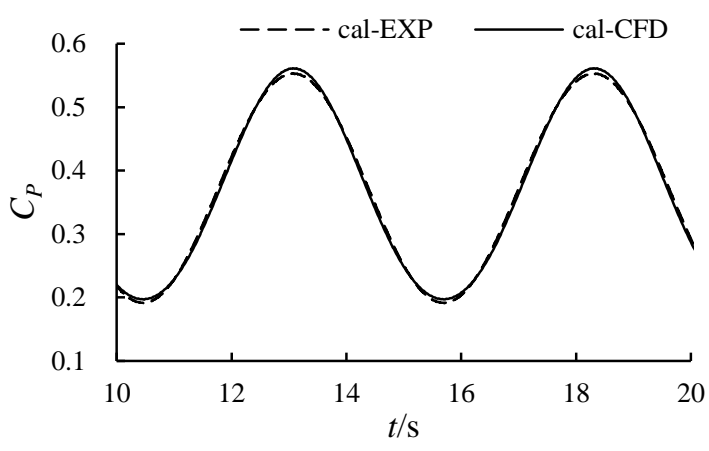

(b) Power coefficient

Fig. 10 Comparison between CFD calculation value and fitting formula calculation value with rotation and surging coupled motion

\subsection{Fitting analysis of the hydrodynamic load in rotation and rolling coupled motion}

The hydrodynamic load fitting formula of the horizontal-axis turbine under the condition of rotation and single degree of freedom swaying motion, which is established in section 2, and the CFD numerical simulation results of the turbine rotation and rolling coupled motion, based on least squares method theory, can be used to solve various coefficients of the fitting formula. The basic parameters of rolling motion are defined as follows

Dimensionless roll angular speed: $\bar{\omega}_{h y}=\frac{A_{h y} \omega_{h y} \cos \left(\omega_{h y} t\right) h_{h y}}{U}$

Dimensionless roll angular acceleration: $\bar{a}_{h y}=-\frac{A_{h y} \omega_{h y}{ }^{2} \sin \left(\omega_{h y} t\right) h_{h y}{ }^{2}}{U^{2}}$

Axial load coefficient progression:

$$
\begin{aligned}
C_{Z} & =C_{Z h y}^{F}+n_{F h y} \bar{\omega}_{h y}+m_{F h y} \bar{a}_{h y} \\
& =C_{Z h y}^{0}+\sum_{k=1}^{\infty} C_{Z h y}^{k} \sin \left(k N \theta(t)+\psi_{F z d}^{k}\right) \\
& +\left\{n_{F h y}^{0}+\sum_{k=1}^{\infty}\left[n_{F h y}^{k} \sin \left(k N \theta(t)+\psi_{F u h y}^{k}\right)\right]\right\} \bar{\omega}_{h y} \\
& +\left\{m_{F h y}^{0}+\sum_{k=1}^{\infty}\left[m_{F h y}^{k} \sin \left(k N \theta(t)+\psi_{F a h y}^{k}\right)\right]\right\} \bar{a}_{h y}
\end{aligned}
$$

Power coefficient progression: 


$$
\begin{aligned}
C_{P} & =C_{P h y}^{F}+n_{P h y} \bar{\omega}_{h y}+m_{P h y} \bar{a}_{h y} \\
& =C_{P h y}^{0}+\sum_{k=1}^{\infty} C_{P h y}^{k} \sin \left(k N \theta(t)+\psi_{P z d}^{k}\right) \\
& +\left\{n_{P h y}^{0}+\sum_{k=1}^{\infty}\left[n_{P h y}^{k} \sin \left(k N \theta(t)+\psi_{P u h y}^{k}\right)\right]\right\} \bar{\omega}_{h y} \\
& +\left\{m_{P h y}^{0}+\sum_{k=1}^{\infty}\left[m_{P h y}^{k} \sin \left(k N \theta(t)+\psi_{P a h y}^{k}\right)\right]\right\} \bar{a}_{h y}
\end{aligned}
$$

where $h_{h y}$ expresses the distance between the turbine rotation axis and the centre of the roll.

Utilizing least squares fitting on power coefficients of different frequencies and axial load coefficients, the various coefficients of the fitting formula can be calculated, as shown in Table 5 and Table 6; only the constant and first-order terms are given in the tables.

Table $5 C_{Z}$ expansion coefficient table (rolling amplitude 15 degrees, rotational speed $230 \mathrm{r} / \mathrm{min}$ )

\begin{tabular}{cccccccccc}
\hline$\omega_{h y}$ & $C_{\text {Zhy }}^{0}$ & $C_{\text {Zhy }}^{1}$ & $\psi_{\text {Fhy }}^{1}$ & $n_{\text {Fhy }}^{0}$ & $n_{\text {Fhy }}^{1}$ & $\psi_{\text {Fuhy }}^{1}$ & $m_{\text {Fhy }}^{0}$ & $m_{\text {Fhy }}^{1}$ & $\psi_{\text {Fahy }}^{1}$ \\
\hline 0.4 & 0.853 & 0.0007 & -62.60 & 0.051 & 0.0031 & -16.45 & 0.091 & 0.0149 & -104.44 \\
0.8 & 0.850 & 0.0003 & -138.68 & 0.056 & 0.0019 & -74.48 & 0.051 & 0.0048 & -35.76 \\
1.2 & 0.851 & 0.0003 & -130.10 & 0.061 & 0.0023 & -131.19 & 0.044 & 0.0028 & 135.16 \\
1.6 & 0.853 & 0.0004 & -47.44 & 0.065 & 0.0025 & -40.80 & 0.036 & 0.0023 & 174.18 \\
2.0 & 0.858 & 0.0004 & -174.45 & 0.071 & 0.0019 & 159.64 & 0.023 & 0.0023 & -4.54 \\
\hline \multicolumn{7}{c}{ Table $6 C_{P}$} & expansion coefficient table (rolling amplitude 15 degrees, rotational speed 230 r/min) \\
\hline$\omega_{h y}$ & $C_{\text {Phy }}^{0}$ & $C_{\text {Phy }}^{1}$ & $\psi_{\text {Phy }}^{1}$ & $n_{\text {Phy }}^{0}$ & $n_{\text {Phy }}^{1}$ & $\psi_{\text {Puhy }}^{1}$ & $m_{\text {Phy }}^{0}$ & $m_{\text {Phy }}^{1}$ & $\psi_{\text {Pahy }}^{1}$ \\
\hline 0.4 & 0.370 & 0.0007 & -63.82 & -0.089 & 0.0029 & -7.00 & 0.086 & 0.0149 & -101.16 \\
0.8 & 0.368 & 0.0006 & -58.14 & -0.096 & 0.0027 & -9.18 & 0.077 & 0.0063 & -117.40 \\
1.2 & 0.368 & 0.0005 & -162.67 & -0.080 & 0.0023 & -162.33 & 0.040 & 0.0012 & 98.27 \\
1.6 & 0.370 & 0.0005 & -34.41 & -0.073 & 0.0024 & 0.40 & 0.025 & 0.0023 & -164.78 \\
2.0 & 0.375 & 0.0006 & -22.64 & -0.068 & 0.0022 & 5.01 & 0.023 & 0.0021 & 149.41 \\
\hline
\end{tabular}

Table 5 and Table 6 show that the values of $C_{\text {Zhy }}^{0}$ and $C_{P h y}^{0}$ are basically stable, and they match $C_{Z}$ and $C_{P}$ when the turbine generates only rotation motion. According to the correlation coefficients in Table 5 and Table 6 , the axial load coefficient and power coefficient when the turbine generates rotation and rolling coupled motion can be calculated by eq. (16) and (17). To verify the accuracy of the fitting formula, calculate the axial load coefficient and power coefficient when the rolling frequency is $1.2 \mathrm{rad} / \mathrm{s}$, and compare it with the CFD calculation value, as shown in Figure 11. Cal-CFD is the CFD calculation value, and cal-EXP is the calculation value by the fitting formula. Figure 11 shows that the CFD calculation value is basically the same as the calculation value by the fitting formula, and this verifies the accuracy of the fitting formula.

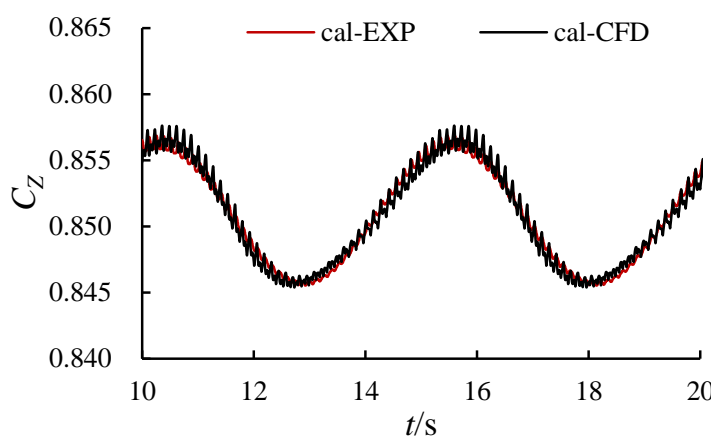

(a) Axial force coefficient

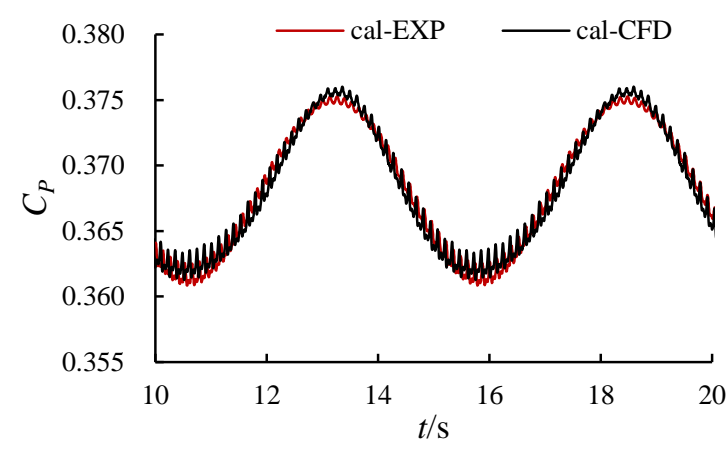

(b) Power coefficient

Fig. 11 Comparison between CFD calculation value and fitting formula calculation value with rotation and rolling coupled motion 


\subsection{Calculation formula of hydrodynamic load in rolling and surging coupled motion}

From the fitting coefficient table, the power coefficient and axial load coefficient calculation values when the turbine generates rotation and surging coupled motion or rotation and rolling coupled motion consist of two parts. One part is the average value, which is basically the same as the value when the turbine only generates rotation motion. The other part is a fluctuation that periodically changes with surging motion or rolling motion. Hence, to foreshadow hydrodynamic research on the horizontal-axis turbine generating more multi-degree of freedom motion, the paper studies the power coefficient and axial load when turbine generates the rotation, rolling and surging coupled motion and resolves them into three parts as follows: 1) the calculation value with only rotation motion; 2) the fluctuation value, which is related to surging motion; and 3) the fluctuation value, which is related to the rolling motion. The calculation value when the turbine generates only rotation motion is easy to obtain; it has been calculated when the calculation value is compared with the experimental value in section 4 of this paper. The fluctuation value that is related to the surging motion can be calculated by eq. (12) and (13), and the fluctuation value that is related to the rolling motion can be calculated by eq. (16) and (17). The axial load coefficient and power coefficient calculation formulas when the turbine generates rotation, rolling and surging coupled motion can be obtained as follows.

$$
\begin{aligned}
C_{Z} & =C_{Z}^{F}+n_{F z d} \bar{u}_{z d}+m_{F z d} \bar{a}_{z d}+n_{F h y} \bar{\omega}_{h y}+m_{F h y} \bar{a}_{h y} \\
& =C_{Z}^{F} \\
& +\left\{n_{F z d}^{0}+\sum_{k=1}^{\infty}\left[n_{F z d}^{k} \sin \left(k N \theta(t)+\psi_{F u z d}^{k}\right)\right]\right\} \bar{u}_{z d} \\
& +\left\{m_{F z d}^{0}+\sum_{k=1}^{\infty}\left[m_{F z d}^{k} \sin \left(k N \theta(t)+\psi_{F a z d}^{k}\right)\right]\right\} \bar{a}_{z d} \\
& +\left\{n_{F h y}^{0}+\sum_{k=1}^{\infty}\left[n_{F h y}^{k} \sin \left(k N \theta(t)+\psi_{F u h y}^{k}\right)\right]\right\} \bar{\omega}_{h y} \\
& +\left\{m_{F h y}^{0}+\sum_{k=1}^{\infty}\left[m_{F h y}^{k} \sin \left(k N \theta(t)+\psi_{F a h y}^{k}\right)\right]\right\} \bar{a}_{h y} \\
C_{P} & =C_{P}^{F}+n_{P z d} \bar{u}_{z d}+m_{P z d} \bar{a}_{z d}+n_{P h y} \bar{\omega}_{h y}+m_{P h y} \bar{a}_{h y} \\
& =C_{P}^{F} \\
& +\left\{n_{P z d}^{0}+\sum_{k=1}^{\infty}\left[n_{P z d}^{k} \sin \left(k N \theta(t)+\psi_{P u z d}^{k}\right)\right]\right\} \bar{u}_{z d} \\
& +\left\{m_{P z d}^{0}+\sum_{k=1}^{\infty}\left[m_{P z d}^{k} \sin \left(k N \theta(t)+\psi_{P a z d}^{k}\right)\right]\right\} \bar{a}_{z d} \\
& +\left\{n_{P h y}^{0}+\sum_{k=1}^{\infty}\left[n_{P h y}^{k} \sin \left(k N \theta(t)+\psi_{P u h y}^{k}\right)\right]\right\} \bar{\omega}_{h y} \\
& +\left\{m_{P h y}^{0}+\sum_{k=1}^{\infty}\left[m_{P h y}^{k} \sin \left(k N \theta(t)+\psi_{P a h y}^{k}\right)\right]\right\} \bar{a}_{h y}
\end{aligned}
$$

the turbine generates only rotation motion.

According to the fitting coefficient table and calculation formulas (18) and (19), we can calculate the axial load coefficient and power coefficient when the turbine generates rotation, rolling and surging coupled motion. Additionally, to verify the accuracy of the calculation formulas, we calculate the axial load coefficient and power coefficient when the turbine generates rotation, rolling and surging coupled motion and compare them with the 
CFD calculation values, as shown in Figure 12. Zd and hy are surging and rolling, respectively. The number means frequency (unit: rad/s); CFD means CFD calculation value; and EXP means the calculation value of the calculation formula.

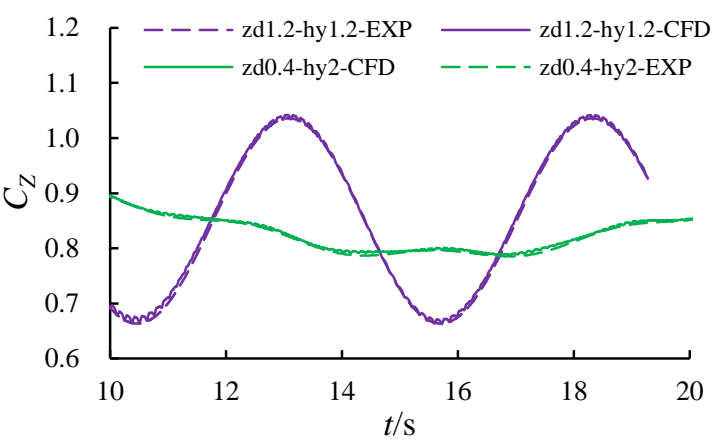

(a) Axial force coefficient

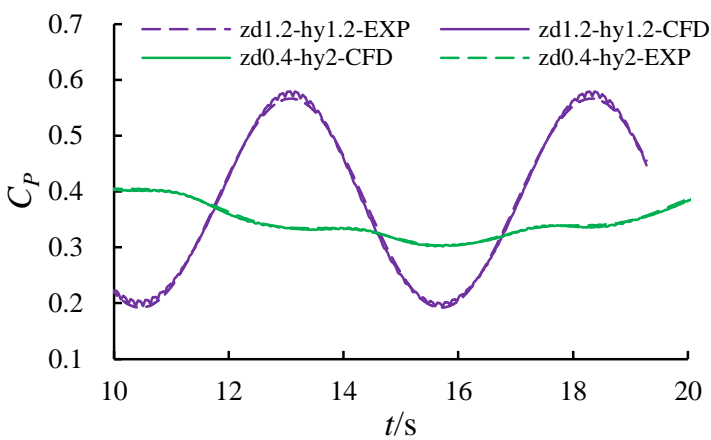

(b) Power coefficient

Fig. 12 Comparison between CFD calculation value and fitting formula calculation value based on rotation, rolling and surging coupled motion (rotational speed $230 \mathrm{r} / \mathrm{min}$ )

It can be seen from the Figure 12 that the CFD calculation values for the axial load coefficient and power coefficient are basically the same as the values of the calculation formula, which verifies the accuracy of the calculation formula. Hence, when forecasting the hydrodynamic load (axial load coefficient and power coefficient) of the horizontal-axis turbine under multi-degree motion, we can first calculate the hydrodynamic load when the turbine generates rotation and the single freedom degree among multi-degree of freedom motion and then obtain the coefficient table by formula (5) according to the hydrodynamic load time-varying curves; finally, the approximate value of the hydrodynamic load when the turbine generates multi-degree of freedom motion can be obtained by the calculation formulas such as (18) and (19). To further verify the accuracy of the method, we will calculate the hydrodynamic load when the turbine generates more multi-degree of freedom motion.

\section{Conclusion}

The CFD method is used to analyse the hydrodynamic characteristics of a horizontal-axis turbine with multiple-degree of freedom of rotation, rolling and surging coupled motion under uniform flow, and the result is compared with the hydrodynamic loads of turbines with rotation and surging coupled motion and rotation and rolling coupled motion. We also analysed the CFD calculation value when the turbine only generates rotation and surging coupled motion or rotation and rolling coupled motion. The results demonstrate the following:

(1) When a turbine generates rolling and surging co-frequency coupled motion, the power coefficient and axial load coefficient will be time-fluctuating, with the fluctuation frequency in accordance with the surging frequency and the fluctuation amplitude increasing with the surging frequency. This will affect the structure safety of the turbine, fatigue life and the steady-state output of the tidal current power station, so the design of the structure and electric control system should pay attention to it;

(2) When the turbine generates rolling and surging different frequency coupled motion, the change regulation of the hydrodynamic load is mainly caused by the surging motion. However, when the rolling frequency is much higher than the surging frequency, the effect of the rolling motion will be reflected. In other words, the hydrodynamic load not only has a fluctuation based on the surging frequency but also includes a fluctuation based on the rolling frequency;

(3) The calculation formulas of the axial load coefficient and power coefficient have been obtained when the turbine generates multiple-degree of freedom (rotation, rolling and surging) coupled motion, and the accuracy of the formula has been verified. They can benefit research on the hydrodynamic load when the turbine generates multi-degree freedom motion. 


\section{Acknowledgement}

This work was supported by the National Project of Special Fund for Marine Renewable Energy (No. GHME2010GC02). Project supported by the National Science Foundation (No.51179077, No.51209060, No.51309125, No.51409128). It is also supported by the University Natural Science Research Project of Jiangsu Province (Grant No. 14KJB570001). Also we give thanks to associate professor Sheng Qi-hu, we revised the program with his help.

\section{Reference}

[1] ZHANG Liang, LI Xin-zhong, GENG Jing, et al. Tidal Current Energy Update 2013 [J]. ADVANCES IN NEW AND RENEWABLE ENERGY, 2013, 1(1): 53-68.

[2] Chen Cun-fu. Study on Blade Optimization and Hydrodynamic Performance of Horizontal Axis Marine Current Turbine[D]. Qingdao, Ocean University of China, 2012.

[3] Ju Hyun Lee, Sunho Park, Dong Hwan Kim, et al. Computational methods for performance analysis of horizontal axis tidal stream turbines [J]. Applied Energy, 2012, 98: 512-523.

[4] Batten W, Bahaj A S, Molland A F, et al. The prediction of the hydrodynamic performance of marine current turbines [J]. Renewable energy. 2008, 33(5): 1085-1096.

[5] A.S. Bahaj, A.F. Molland, J. R. Chaplin, et al. Power and thrust measurements of marine current turbines under various hydrodynamic flow conditions in a cavitation tunnel and a towing tank [J]. Renewable energy, 2007, 32(3): 407-426.

[6] L.E. Myers, A.S. Bahaj. Experimental analysis of the flow field around horizontal axis tidal turbines by use of scale mesh disk rotor simulators [J]. Ocean Engineering, 2010, 37: 218-227.

[7] W.M.J. Batten, A.S. Bahaj, A.F. Molland, J.R. Chaplin, Hydrodynamics of marine current turbines, Renewable Energy, 31 (2006) 249-256.

[8] A.S. Bahaj, A.F. Molland, J.R. Chaplin, W.M.J. Batten, Power and thrust measurements of marine current turbines under various hydrodynamic flow conditions in a cavitation tunnel and a towing tank, Renewable Energy, 32 (2007) 407-426.

[9] F. Maganga, G. Germain, J. King, G. Pinon, E. Rivoalen, Experimental characterisation of flow effects on marine current turbine behaviour and on its wake properties, IET. Renew. Power Gener. 4 (6) (2010) 498-509.

[10] Barltrop N, Varyani KS, Grant A, et al. Investigation into wave current interactions in marine current turbines[J]. ProcIMech E Part A e J Power Energy. 2007, 42:221-233P.

[11] Galloway PW, Myers LE, Bahaj AS. Studies of scale turbine in close proximity to waves[C]. In: 3rd international conference on ocean energy, 2010.

[12] Luksa Luznik, Karen A. Flack, Ethan E. Lust Katharin Taylor. The effects of surface waves on the performance characteristics of a model tidal turbine[J]. Renewable energy. 2013, 58:108-114P.

[13] Ethan E. Lust, Luksa Luznik, Karen A. Flack, et al. The influence of surface gravity waves on marine current turbine performance[J]. Marine Energy. 2014, 3(4):27-40P.

[14] Ma Yong, Zhang Liang, Sheng Qi-hu, et al. The test study on hydrodynamic characteristics of floating tidal power generation devices $[\mathrm{J}]$. Journal of huazhong university of science and technology (natural science edition), 2012, 10(40): 123-127.

[15] Zhang Liang, Wang Shuqi, Sheng Qihu, Jing Fengmei, Ma Yong. The effects of surge motion of the floating platform on hydrodynamics performance of horizontal-axis tidal current. Renewable Energy, 2015, 74: 796-802.

[16] WANG Shu-qi, XIAO Gang, ZHANG Liang, et al. Supporting column influence analysis of horizontal axis tidal current turbine [J]. Journal of huazhong university of science and technology (natural science edition), 2014, 42(4): 81-85. 